\title{
Antibacterial and Antioxidant Activity of Metabolites From Bioconverted Docosahexaenoic Acid Using Gut Bacteria
}

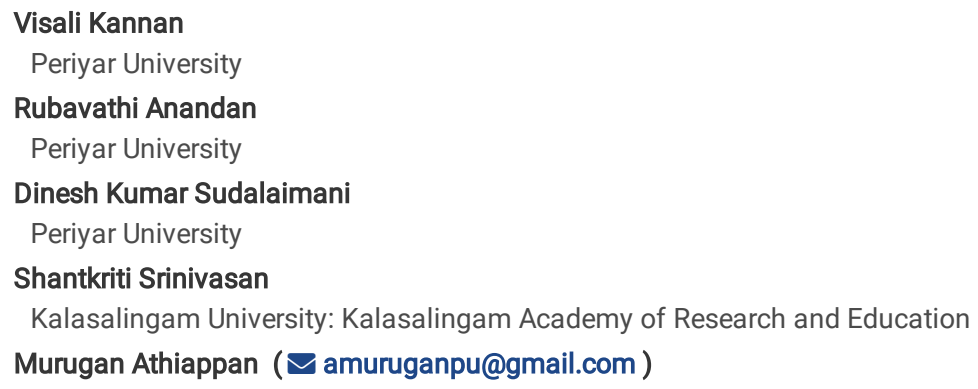

Research Article

Keywords: Docosahexanoic acid, Gut Bacteria, Bioconversion, Antimicrobial activity.

Posted Date: July 16th, 2021

DOI: https://doi.org/10.21203/rs.3.rs-674393/v1

License: (c) (7) This work is licensed under a Creative Commons Attribution 4.0 International License. Read Full License 


\begin{abstract}
Docosahexaenoic acid (DHA) is an essential fatty acid necessary for brain development in both infants and adults. However, the role of gut microbiome and their metabolites produced from DHA remain unclear. In present study, the bacterial isolates Lactobacillus spp. Clostridium spp. Escherichia coli;

Staphylococcus spp. Enterococcus spp. were used to convert the metabolites from DHA with SM medium supplemented with $200 \mathrm{mg}$ of DHA as substrate. The metabolites were extracted after 24 hours of incubation at $37^{\circ} \mathrm{C}$ and analyzed by GC/MS. The antimicrobial activity of these metabolites confirmed their effectiveness against clinical pathogens such as Bacillus cereus, Escherichia coli, Pseudomonas aeruginosa, Salmonella enteritidis and Staphylococcus aureus.
\end{abstract}

\title{
Introduction
}

DHA (docosahexaenoic acid) is an important nutrient needed during critical life stages (such as lactation) and for immunity [1]. It is vital during pregnancy and infancy because it plays a critical role in the brain development [2]. DHA accounts for over $90 \%$ of the omega-3 fatty acids in human brain, as well as up to $25 \%$ of its overall fat content.It is primarily found in cell membranes, where it improves the fluidity of the membranes and gaps between cells. This allows nerve cells to transmit and receive electrical signals more easily [3]. Therefore, adequate levels of DHA appear to make nerve cell communication simpler, faster, and more effective. Low levels in the brain or eyes may cause communication between cells to slow down, resulting in blurry vision or impaired cognitive function.DHA is essential for brain tissue growth and function, especially during infancy and development [4]. For the eyes and brain to grow normally, it accumulates in the central nervous system. The baby's DHAlevels are determined by its ingestion during the third trimester of pregnancy, with the largest accumulation occurring in the brain during the first few months of life [5]. Antibacterial activity is exhibited by DHA derivatives through a variety of mechanisms, all of which mainly involve bacterial cell membrane perturbation.DHA has anti-inflammatory properties and prevents tumorigenesis, in addition to its antimicrobial and antiviral properties $[6,7,8,9]$. Following cerebral ischemia, the nerve cell membrane releases DHA, which induces oxidative stress and neurotrophin activation, and is metabolized to NPD1-like DHA, which prevents ischemic nerve cell death. These findings suggested that DHA combined with NPD1 could prevent ischemic neuronal damage[10].

Later, DHA having a higher unsaturation level than EPA, was demonstrated to have an inhibitory effect on gram negative bacteria that surpasses that of EPA [11]. Long chain fatty acids are well known to be inhibitory on gram positive bacteria even at low concentrations. However, gram negative bacteria are known for their complex lipopolysaccharide layer as compared to the gram positive bacteria. But, PUFA are known to have inhibitory effect on these strains as compared to saturated fatty acids as they are readily incorporated into the outer cell membranes of these organisms, where they significantly increase membrane fluidity. It is possible that by opening up permeability channels, the concentration gradients necessary between the organism and its environment may be dissipated resulting in fatality of the organism[12].Among these, DHA is one of the most effective fatty acid compounds. In addition to its documented antimicrobial and antiviral properties, DHA possesses anti-inflammatory activity and inhibit tumorigenesis [6, 7, 8, 9]. Although microbial bioconversion of EPA and DHA was reported but the antimicrobial activity of bioconversion products have not been investigated so far[11]. The microbicidal activity of selected LCUFAs and their derivatives has been reported on various enveloped viruses, parasitesand pathogenic bacteria such as Pseudomonas aeruginosa, Bacillus subtilis, Listeria monocytogenes, Helicobacter pylori, Staphylococcus aureus and Neisseria gonorrhea[6, 11]. Fish oil decreases the proliferation of tumor cells, whereas arachidonic acid, a long chain n-6 fatty acid, increases their proliferation [13]. Therefore, in this study, we investigated whether the antimicrobial properties of DHA derivatives against clinical isolates, thereby supporting their usein the treatment of chronic infection in patients.

\section{Materials And Methods}

\section{Growth Media}

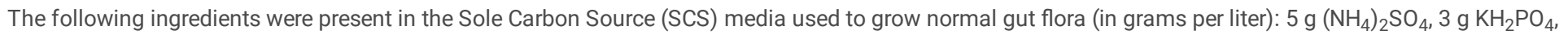
$0.5 \mathrm{~g} \mathrm{MgSO}_{4} 7 \mathrm{H}_{2} \mathrm{O}, 15 \mathrm{mgEDTA}, 4.5 \mathrm{mg} \mathrm{ZnSO}_{4} 7 \mathrm{H}_{2} \mathrm{O}, 4.5 \mathrm{mg} \mathrm{CaCl} 2 \mathrm{H}_{2} \mathrm{O}, 3 \mathrm{mg} \mathrm{FeSO}{ }_{4} 7 \mathrm{H}_{2} \mathrm{O}, 1 \mathrm{mg} \mathrm{MnCl} .4 \mathrm{H}_{2} \mathrm{O}, 1 \mathrm{mg} \mathrm{H}_{3} \mathrm{BO}_{3}, 0.4 \mathrm{mg} \mathrm{Na}_{2} \mathrm{MoO}_{4} .2 \mathrm{H}_{2} \mathrm{O}, 0.3 \mathrm{mg}$ $\mathrm{CuSO}_{4} \cdot 5 \mathrm{H}_{2} \mathrm{O}, 0.3 \mathrm{mgCoCl}_{2} \cdot 6 \mathrm{H}_{2} \mathrm{O}$ and $0.1 \mathrm{mg} \mathrm{Kl}$; The $\mathrm{pH}$ wasadjusted to 5.5 using $\mathrm{HCL}$, and the media was sterilized through a $0.22 \mu \mathrm{m}$ filter. Solid medium was prepared byadding $15 \mathrm{~g}$ agar per liter of liquid SCS media followed byautoclaving at $121^{\circ} \mathrm{C}[14]$.

\section{Fecal Sample Collection}

The stool samples were taken from four healthy volunteers ranging in age from 20 to 75 years' old who lived in the same neighborhood. Within $15 \mathrm{~h}$ of collection, samples were sent to the researchers, packed with parafilm, then transported and stored in ice.

\section{Isolation and Characterization of Gut Microbial Flora from Human Fecal Sample}

Approximately $125 \mathrm{mg}$ of fecal material was dissolved in $5 \mathrm{~mL}$ of SCS media. To avoid the transfer of residual alternative carbonsources present in the original inoculum, samples were transferred $(2.5 \mu \mathrm{L})$ into fresh SCS media $(5 \mathrm{~mL})$ and incubated at $37^{\circ} \mathrm{C}$ for $24-48 \mathrm{~h}$. The isolatesfrom the liquid cultures were obtained by plating cultures on an SCS agar medium plate and incubated at $37^{\circ} \mathrm{C}$ for $24-48 \mathrm{~h}[14]$. The singlecolonies were picked and re-streaked on SCS plates and further isolates were identified by morphological and biochemical characteristics.

\section{Bioconversion of Docosahexaenoic Acid}

Bioconversion was described previously[11] and carried out in five set $50 \mathrm{~mL}$ of SM broth with supplement of DHA tablet which contains $200 \mathrm{mg}$ of DHA were added to $24 \mathrm{~h}$ old cultures of five different gut bacteria to the five set of SM broth individually and followed by continued incubation for an $24 \mathrm{~h}$ at $37^{\circ} \mathrm{C}$ and bioconversion was allowed to proceed

\section{Extraction of Fatty Acids from Bio-converted Broth}


Bio-converted broth were suspended in $3 \mathrm{~mL}$ of $4 \mathrm{molL}^{-1}$ sodium hydroxide, and incubated at $90^{\circ} \mathrm{C}$ for $90 \mathrm{~min}$. After cooling, the pH of the sample was adjusted to 2 with Hydrochloric acid. Fatty acids were then extracted by adding $2 \mathrm{~mL}$ anhydrous diethyl ether and separated by centrifugation at $5500 \times \mathrm{g}$ for $10 \mathrm{~min}$. The upper phase was removed and dehydrated by adding anhydrous sodium sulfate. The dehydrated fatty acids were collected and dried under a stream of nitrogen. Next, $50 \mu$ Lbistrimethylsilyltrifluoroacetamide (BSTFA) was added, and the mixture was incubated at $70^{\circ} \mathrm{C}$ for 30 min and dried under a stream of nitrogen. The fattyacids were dissolved in $100 \mu \mathrm{L}$ hexane for GC/MS analysis [15].

\section{GC-MS Analysis}

Fatty acid composition analysis was performed on the Shimadzu GCMS QP 2020 that employed a fused silica column, packed with SH-Rxi-\%Sil MS (30 m $\times$ $0.25 \mathrm{~mm} \mathrm{ID} \times 250 \mu \mathrm{mdf}$ ) and the components were separated using Helium as carrier gas at a constant flow of $1 \mathrm{ml} / \mathrm{min}$. The injector temperature was set at $280^{\circ} \mathrm{C}$ during the chromatographic run. $1 \mu \mathrm{L}$ of extract sample injected into the instrument the oven temperature was as follows: $40^{\circ} \mathrm{C}(2 \mathrm{~min})$; followed by $280^{\circ} \mathrm{C}$ at the rate of $10^{\circ} \mathrm{C} \mathrm{min}-1$ and $280^{\circ} \mathrm{C}$, where it was held for $3 \mathrm{~min}$. The mass detector conditions were: transfer line temperature $280^{\circ} \mathrm{C}$; ion source temperature $230^{\circ} \mathrm{C}$; and ionization mode electron impact at $70 \mathrm{eV}$, a scan time $0.2 \mathrm{~s}$ and scan interval of $0.1 \mathrm{~s}$. The fragments from 40 to $550 \mathrm{Da}$. The spectrums of the components were compared with the database of spectrum of known components stored in the GC-MS NIST (2017) library.

\section{Antibacterial activity of bio-converted DHA extract}

The antimicrobial activity of the compound was determined using agar-well diffusion method. The microorganisms Bacillus cereus, Escherichia coli, Pseudomonas aeruginosa, Salmonella enteritidis and Staphylococcus aureus were obtained from the SKS Clinical Laboratory, Salem, Tamil Nadu, India. The Gentamycin containing discs (Nam KhoaBioTek Company, Vietnam) $(10 \mu \mathrm{g} / \mathrm{mL}$ ) were used as positive controls and negative controls were sterilized distilled water containing paper discs. $900 \mu \mathrm{g}$ of the bio-converted DHA extract was weighed and dissolved in 10mL Dimethyl sulfoxide (DMSO) to obtain a concentration of $90 \mu \mathrm{g} / \mathrm{mL}$ of the extract; this was the initial concentration of the extract used to determine the antimicrobial activity of the extract. Mueller Hinton agar was prepared by following the medium sterilized at $121^{\circ} \mathrm{C}$ for $15 \mathrm{~min}$; the sterilized medium was then poured into sterile petri dishes. The plates were allowed to cool and solidify. The sterilized medium was seeded with $0.1 \mathrm{~mL}$ of the standard inoculum was spread evenly over the surface of medium with a sterile swab. Wells were bored into the solidified inoculated using a standard broth borer of $6 \mathrm{~mm}$ in diameter. $0.1 \mathrm{~mL}$ of the solution of the extract of concentration $90 \mu \mathrm{g} / \mathrm{mL}$ was then introduced into each well on the medium. The inoculated medium was then incubated at $37^{\circ} \mathrm{C}$ for $24 \mathrm{~h}$ after which each plate was observed for the zone of inhibition of growth which was measured with a transparent ruler and the result recorded in millimeters.

\section{Results}

\section{Isolation and Characterization of Gut Microbial Flora from Human Fecal sample}

There were 65 colonies from the human fecal sample. Based on colony morphology, 16 bacteria wereselected for further biochemical characteristics [Table 1] which was defined by Bergey's Manual. Among 16 bacterial isolate screened from colony morphological characteristics, Lactobacillus spp. Clostridium spp., Escherichia coli, Staphylococcus spp. Enterococcus spp. are thegut bacteria which was predominantly present in both normal human intestine and the disease human intestine[16]. These five gut bacteria were further used for bioconversion of Docosahexaenoic acid.

\section{Bioconversion of DHA using Gut bacteria and GC-MS Analysis of Metabolites from Bio- converted DHA extract}

After $24 \mathrm{~h}$, bioconvertedDocosahexanoic acid broth was subjected to fatty acid extraction. The crude extract was further analyzed for GC-MS analysis and antibacterial activity. The Normal Gut flora which was isolated from human fecal converted Docosahexaenoic acid into various therapeutic metabolites analyzed by GC-MS analysis and it has various medicinal properties are tabulated [Fig. 1, Table 2, Table 3, Table 4, Table 5, and Table 6].

\section{Anti-bacterial activity of crude extract from bioconverted DHA}

From the GC-MS analysis, compounds from bioconverted DHA have varies medicinal properties and most of the compounds have similar property of antimicrobial activity. To ensure that we evaluate the antibacterial activity for their extract. The antibacterial activity of bioconverted DHA extract was presented in [Table 7]. Among 5 tested microorganisms, the extract featured the strongest antimicrobial effect of Bifidobacterium spp., Eschericia coli, Citrobacter spp. Bioconverted DHA extractagainst B. cereus with the diameter of inhibition zone about 25mm, 26mm, 24mm. Bifidobacterium spp., Lactobacillus spp.,Eschericiacoli,Citrobacterspp.,Enterobacter spp.bioconverted DHA extract showed activity againstStaphylococcus aureus (20mm, 19mm, $18 \mathrm{~mm}, 20 \mathrm{~mm})$, Escherichia coli(15mm, 16mm, 14mm, 15mm, 17mm), Pseudomonas aeruginosa (19mm, 18mm, 20mm, 19mm, 20mm), and Salmonella enteritidis $(16 \mathrm{~mm}, 15 \mathrm{~mm}, 14 \mathrm{~mm}, 15 \mathrm{~mm}, 13 \mathrm{~mm})$. There was no activity found against the DMSO and distilled water. The findings suggest the potential application of bioconverted DHA extract as the antimicrobial agent, because the diameter of inhibition zone of extract were near to that of positive controls.

\section{Discussion}

Lactobacillus spp., Clostridium spp., Escherichia coli, Staphylococcus spp.,andEnterococcus spp. have multiple roles in the human body which stabilize the gastric acid, hepatic bile and digestive enzymes of gastrointestinal tract and it can modulate gut microbiota and microbiota associated metabolic pathways [17].These gut bacteria are predominantly present in both the healthy and diseased person [16]. Therefore, these microbes were used in invivostudy to evaluate the relationship between the gut bacteria and DHA. These gut bacteria converted DHA into 82 metabolites [Table 8] which have various therapeutic properties like anti-oxidant, anti-bacterial, anti-depressant, anti-tumor, anti-biofilm, anti-melanogenic, anxiolytic effects, anti-obesity, anti-diarrheal, anti-fungal, anti-cancer, anti-inflammatory, alpha-amylase inhibitory, anti-staphylococcal activity, pancreatic lipase inhibitory activity, and immunotherapeutic agent. 
Commonly, Lactobacillus spp., Clostridium spp., Escherichia coli, Staphylococcus spp., Enterococcus spp. are present in healthy person[18] but decrease if the person is said to be in a diseased state[19]. This could lead to many disorders like depression and other hormonal changes due to the lack of the therapeutic metabolites. We suggest that to improve the role of these therapeutic metabolites in the diseased person, it should be given to them as a combination of DHA and these microbes as probiotics which can increase the normal flora of intestine.

The most common activity among all metabolites obtained from bioconverted DHA extract is the anti-bacterial activity. Hence, in this studythe crude extract was evaluated against clinical pathogens and it showed effective and strong antibacterial activity against them. In an earlier study, DHA was bioconverted using $P$. aeruginosa PR3 and the crude extract showed effective antibacterial activity against four gram-positive bacteria, Bacillus subtilis, Listeria monocytogenes, Staphylococcus aureus (ATCC 6538) and S. aureus (KCTC 1916) and seven gram-negative bacteria, Enterobacteraerogenes, Escherichia coli, E. coli 0157:H7, Pseudomonas aeruginosa, Salmonella enteritidis and S. typhimurium[11]. Whereas, we analyzed the bioconverted DHA with Lactobacillus spp., Clostridium spp., Escherichia coli, Staphylococcus spp., Enterococcus spp. crude extract which showed aneffective antibacterial activity against the clinical pathogens B. cereus, E. coli, P. aeruginosa, S. typhimurium, S. aureus and S. enteritidiswhen compared to the standard antibiotic Gentamycin.

\section{Conclusion}

From this work, itcan be concluded that metabolites from extract of bioconverted DHA with Lactobacillus spp., Clostridium spp., Escherichia coli, Staphylococcus spp., Enterococcus spp. has many medicinal properties such as antibacterial, antioxidant, anxiolytic, phytotoxicity and antiviral activities. This work reported for the first time the metabolite from this bioconversion to possess an anti-depressant effect. In conclusion, Normal flora of gut can convert the Docosahexaenoic acid into the various therapeutic metabolites.

\section{Declarations}

Ethics Approval: Not Applicable.

Consent to Participate: Authors agreed to participate in this research.

Consent for Publication: All the authors have approved the last version of the manuscript for its submission/publication.

Author Contribution: V.K and M.A contributed to the study conception and design; V.K conducted experiments; and V.K, R.A, D.K.S, S.S and M.A contributed to the writing of this manuscript and approved the final version.

Funding: Not Applicable.

Competing Interests: The authors declare no competing interests.

Availability of data and material: Not Applicable.

\section{References}

1. Simopoulos, A. P. (1991). Am. J. Clin. Nutr, 54, 438-463

2. Meharban Singh. Indian Journal of Pediatrics, Volume 72.

3. Stilwell, B., Diallo, K., \& Zurn, P. (2003). R, Mario Dal Poz, OrvillAdams and James Buchan. Resources for Health

4. KuratkoC, Nelson, N. B. E. C., \& Salem, N. E. B and Jr.(2013), Nutrients, 5, 2777-2810; doi:10.3390/nu5072777.

5. Guesnet, P., \& Alessandri, J. M. (2011). Biochimie 93, 7-12.

6. BougnouxP. CurrOpin Clin NutrMetab Care, 2 121-126.

7. CalderP.C.Am J Clin Nutr, 831505S-1519S.

8. Calviello, G., Palozza, P., Franceschelli, P., \& Bartoli, G. M. (1997). Lipids, 32, 1075-1083

9. Kang, K. S., Wang, P., Yamabe, N., Fukui, M., Jay, T., \& Zhu, B. T.(2010) PLoS ONE, 5: e10296.

10. Kazuo, Y. (2020). )J. Neurosci. Res, 00, 1-12

11. Shin, S. Y., \& Bajpai, V. K. HakRyul Kim, Sun Chul Kang(2007). Journal of Food Microbiology 113 (2007) 233-236.

12. Thompson, H., Zhu, Z., \& Banni, S. (1997). Kathleen Darcy, Tami Loftus, and Clement Ip. Cancerresearch, 57, 5067-5072. November15

13. Lloyd, A., Horrocks, A., \& Yeo, Y. K(1999). Pharmacological ResearchVolume 40, Issue 3, Pages211-225.

14. Zhao Xin, T., Fengwei, W., Gang, L., \& Xiaoming, Zhang Qiuxiang, Zhang Hao and Chen Wei. (2012)FEMS Microbiol Ecol82703-712.

15. Li, Y., Wu, S., Li, L. W. Y., Shi, F., \& Wang, X. (2010). J Sci Food Agric, 90, 1380-1383

16. Balakrishnan RamakrishnaS. J Clin Gastroenterol Volume 41, Supp. 1.

17. Maity, H. K., Biswas, S., Das, S., Sree Lakshmi, P., \& Samanta, I. (2020). Indian J. Anim. Hlth. 59(2)-Special Issue: 01-12.

18. Huifang Si, Q., Yang, H., Ding, H. C., \& Wang, H., Xuhong Lin. (2021)Seminars in Cancer Biology 70 3-10.

19. Hao Wang, C. X., Wei, L. M., \& Ling-Yun Zhu. (2018). and. Biotechnology \& Biotechnological Equipment, Vol. 32, No. 5, $1075-1080$.

\section{Tables}


Table-1: Morphological and Biochemical Characteristics of Gut Bacteria from fecal sample

\begin{tabular}{|c|c|c|c|c|c|c|c|c|c|c|c|c|}
\hline $\begin{array}{l}\text { Bacterial } \\
\text { Isolate }\end{array}$ & Bacterial Name & $\begin{array}{l}\text { Gram } \\
\text { Staining }\end{array}$ & Shape & Motility & Spore & Catalase & Oxidase & MR & VP & Indole & $\begin{array}{l}\text { Nitrate } \\
\text { Reduction }\end{array}$ & $\begin{array}{l}\text { Fermentation } \\
\text { of Fructose }\end{array}$ \\
\hline $\mathrm{H} 1$ & $\begin{array}{l}\text { Enterococcus } \\
\text { spp. }\end{array}$ & + & Cocci & - & - & - & - & - & + & - & + & + \\
\hline $\mathrm{H} 2$ & $\begin{array}{l}\text { Lactobacillus } \\
\text { spp. }\end{array}$ & + & Rod & - & - & - & - & - & - & - & + & + \\
\hline $\mathrm{H} 3$ & Clostridium spp. & + & Bacilli & + & + & - & - & - & - & - & - & + \\
\hline $\mathrm{H} 4$ & Eschericia coli & - & Bacilli & + & - & + & - & + & - & + & + & - \\
\hline $\mathrm{H} 5$ & Citrobacter spp. & - & Bacilli & + & - & + & - & + & - & - & + & - \\
\hline $\mathrm{H} 6$ & $\begin{array}{l}\text { Enterobacter } \\
\text { spp. }\end{array}$ & - & Rod & + & & + & - & - & + & - & + & - \\
\hline $\mathrm{H7}$ & Proteus spp. & - & Rod & + & & + & - & + & - & + & + & - \\
\hline $\mathrm{H} 8$ & $\begin{array}{l}\text { Streptococci } \\
\text { spp. }\end{array}$ & + & cocci & - & & - & & + & + & - & - & - \\
\hline $\mathrm{H9}$ & Bacillus spp. & + & Bacilli & + & + & + & - & + & + & - & - & + \\
\hline $\mathrm{H} 10$ & $\begin{array}{l}\text { Staphylococcus } \\
\text { spp. }\end{array}$ & + & cocci & & & + & - & + & - & - & & - \\
\hline $\mathrm{H} 11$ & $\begin{array}{l}\text { Bifidobacterium } \\
\text { spp. }\end{array}$ & + & Rod & - & - & - & - & & & - & - & + \\
\hline $\mathrm{H} 12$ & Clostridium spp. & + & Coccobacilli & - & + & - & - & + & - & - & - & + \\
\hline $\mathrm{H} 13$ & Leuconostocspp & + & Cocci & - & & - & - & & & & & - \\
\hline $\mathrm{H} 14$ & $\begin{array}{l}\text { Enterococcus } \\
\text { spp. }\end{array}$ & + & Cocci & - & - & - & - & & & & & - \\
\hline $\mathrm{H} 15$ & $\begin{array}{l}\text { Fusobacterium } \\
\text { spp. }\end{array}$ & - & Rod & - & - & - & - & - & - & + & - & + \\
\hline $\mathrm{H} 16$ & Bacteroides spp. & - & Rod & - & - & + & - & & & - & & - \\
\hline
\end{tabular}

Table-2: Metabolites from bioconverted DHA using Lactobacillus spp. 


\begin{tabular}{|c|c|c|c|c|c|}
\hline S.No & R.Time & $\begin{array}{l}\text { Percentage } \\
\%\end{array}$ & Compound Name & Medicinal Properties & References \\
\hline \multirow[t]{3}{*}{1} & 22.176 & 13.27 & 2-Nonadecanone & Anti-depression & Lee et.al., 2018 \\
\hline & & & & Anti-bacterial & Mihigo et.al., 2015 \\
\hline & & & & Anti-tumor & Figueiredo et.al., 2014 \\
\hline 2 & 21.941 & 12.91 & Z,Z-6,27-Hexatriactontadien-2-one & - & - \\
\hline \multirow[t]{3}{*}{3} & 21.164 & 8.47 & cis-9-Hexadecenal & Anti-biofilm & Hoda et.al., 2019 \\
\hline & & & & Anti-melanogenic & Hoda et.al., 2020 \\
\hline & & & & Anti-fungal & \\
\hline \multirow[t]{3}{*}{4} & 21.395 & 7.89 & Eicosanal- & Anti-depressant & Uddin et.al., 2021 \\
\hline & & & & Anxiolytic effects and & \\
\hline & & & & Anti-oxidant & \\
\hline 5 & 26.623 & 7.54 & Stigmast-5-En-3-Ol, Oleat & Anti-obesity & Ghinmy et.al., 2019 \\
\hline \multirow[t]{2}{*}{6} & 26.922 & 5.07 & Tetrapentacontane & Anti-microbial & Kerem et.al., 2019 \\
\hline & & & & Anti-Oxidant & Xuanji et.al., 2016 \\
\hline \multirow[t]{2}{*}{7} & 22.489 & 3.26 & Oleic Acid & Anti-tumor & Giulitt et.al, 2021 \\
\hline & & & & Anti-Microbial & Ghavam et.al., 2021 \\
\hline \multirow[t]{2}{*}{8} & 22.698 & 3.20 & Octadecanoic acid & Anti-bacterial & Daniels et.al., 2021 \\
\hline & & & & Anti-oxidant & \\
\hline \multirow[t]{3}{*}{9} & 21.275 & 2.15 & (Z)-3-(Heptadec-10-en-1-yl)phenol & Anti-bacterial & Van et.al., 2020 \\
\hline & & & & Anti-diarrheal & Nemkul et.al., 2021 \\
\hline & & & & Anti-oxidant & \\
\hline 10 & 20.193 & 2.69 & 2-Heptadecanone & & \\
\hline \multirow[t]{4}{*}{11} & 20.774 & 2.05 & n-Hexadecanoic acid & Anti-bacterial & Ansari et.al., 2021 \\
\hline & & & & Anti-fungal & \\
\hline & & & & Anti-biofilm & \\
\hline & & & & Anti-cancer & \\
\hline 12 & 24.730 & 1.95 & Heneicosane & Anti-inflammatory & Barda et.al., 2021 \\
\hline \multirow[t]{2}{*}{13} & 15.782 & 1.65 & Phenol, 2,4-Bis(1,1-Dimethylethyl)- & Anti-fungal & Devi et.al., 2021 \\
\hline & & & & Anti-oxidant & Idih et.al., 2021 \\
\hline 14 & 19.326 & 1.64 & Hexadecanal & - & - \\
\hline 15 & 21.880 & 1.34 & 8,11-Heptadecadienal, $(8 Z, 11 Z)-$ & - & - \\
\hline 16 & 21.102 & 1.28 & 9,17-Octadecadienal, (Z)- & Antimicrobial agent & Insanu et.al., 2021 \\
\hline 17 & 23.985 & 0.99 & 2-Pentacosanone & - & - \\
\hline 18 & 16.871 & 0.99 & Hexadecane & - & - \\
\hline 19 & 27.063 & 0.97 & 8-Octadecanone & Antimicrobial metabolite & Tayung et.al., 2011 \\
\hline 20 & 25.397 & 0.89 & $\begin{array}{l}\text { Stigmasta-5,22-Dien-3-Ol, Acetat, (3- } \\
\text { Beta,2 }\end{array}$ & Antimicrobial & Richa Bhardwaj 2018 \\
\hline 21 & 28.040 & 0.72 & 9-Heptadecanone & - & - \\
\hline 22 & 27.786 & 0.70 & Z,Z-6,28-Heptatriactontadien-2-one & $\begin{array}{l}\text { Alpha-amylase Inhibition And Antioxidant } \\
\text { Activity }\end{array}$ & $\begin{array}{l}\text { Unnikrishnan et.al., } \\
2015\end{array}$ \\
\hline
\end{tabular}

Table-3: Metabolites from bioconverted DHA using Clostridium spp. 


\begin{tabular}{|c|c|c|c|c|c|}
\hline S.No & R.Time & Percentage\% & Compound Name & Medicinal Properties & References \\
\hline \multirow[t]{2}{*}{1} & \multirow[t]{2}{*}{22.996} & \multirow[t]{2}{*}{15.86} & \multirow[t]{2}{*}{ Octadecanoic acid } & Anti-bacterial & \multirow[t]{2}{*}{ Daniels et.al., 2021} \\
\hline & & & & Anti-oxidant & \\
\hline 2 & 22.743 & 13.93 & 6-Octadecenoic acid & Anti-bacterial & Al-fekaiki1 et.al., 2017 \\
\hline \multirow[t]{4}{*}{3} & \multirow[t]{4}{*}{20.948} & \multirow[t]{4}{*}{10.92} & \multirow[t]{4}{*}{ n-Hexadecanoic acid } & Anti-bacterial & \multirow[t]{4}{*}{ Ansari et.al., 2021} \\
\hline & & & & Anti-fungal & \\
\hline & & & & Anti-biofilm & \\
\hline & & & & Anti-cancer & \\
\hline \multirow[t]{2}{*}{4} & \multirow[t]{2}{*}{27.000} & \multirow[t]{2}{*}{8.34} & \multirow{2}{*}{$\begin{array}{l}\text { 9-Octadecenoic acid (Z)-, 2,3-dihydroxypropyl } \\
\text { ester }\end{array}$} & Anti-microbial & Kumari et.al., 2021 \\
\hline & & & & Anti-Fungal & Antonio et.al., 2021 \\
\hline \multirow[t]{2}{*}{5} & \multirow[t]{2}{*}{27.214} & \multirow[t]{2}{*}{7.11} & \multirow[t]{2}{*}{ Octadecanoic acid, 2,3-dihydroxypropyl ester } & Anti-microbial & Kumari et.al., 2021 \\
\hline & & & & Anti-oxidant & Vural et.al., 2021 \\
\hline 6 & 25.389 & 4.40 & $\begin{array}{l}\text { 9-Octadecenoic acid (Z)-, oxiranylmethyl } \\
\text { ester }\end{array}$ & - & - \\
\hline 7 & 26.680 & 4.30 & n-Propyl 9-octadecenoate & - & - \\
\hline 8 & 25.582 & 3.50 & Glycidyl palmitate & Anti-staphylococcal activity & Houdkova et.al., 2021 \\
\hline 9 & 21.398 & 11.29 & Eicosanal- & $\begin{array}{l}\text { Anti-depressant Anxiolytic effects and Anti- } \\
\text { oxidant }\end{array}$ & Uddin et.al., 2021 \\
\hline \multirow[t]{3}{*}{10} & \multirow[t]{3}{*}{21.168} & \multirow[t]{3}{*}{3.01} & \multirow[t]{3}{*}{ cis-9-Hexadecenal } & Anti-biofilm & Hoda et.al., 2019 \\
\hline & & & & Anti-melanogenic & \multirow[t]{2}{*}{ Hoda et.al., 2020} \\
\hline & & & & Anti-fungal & \\
\hline \multirow[t]{2}{*}{11} & \multirow[t]{2}{*}{25.490} & \multirow[t]{2}{*}{2.83} & \multirow{2}{*}{$\begin{array}{l}\text { Hexadecanoic acid, 2-hydroxy-1- } \\
\text { (hydroxymethyl)ethy }\end{array}$} & Anti-fungal & Khan et.al., 2021 \\
\hline & & & & Anti-microbial & Kumari et.al., 2021 \\
\hline 12 & 26.786 & 2.31 & 1-Cyclohexyldimethylsilyloxybutane & - & - \\
\hline 13 & 26.880 & 2.26 & Octadecanoic acid, propyl ester & - & - \\
\hline 14 & 24.491 & 1.75 & Eicosanoic acid & Anti-bacterial & Pavani et.al., 2021 \\
\hline \multirow[t]{2}{*}{15} & \multirow[t]{2}{*}{6.560} & \multirow[t]{2}{*}{1.43} & \multirow[t]{2}{*}{ Oxime-, methoxy-phenyl- } & Pancreatic lipase & \multirow[t]{2}{*}{ Anand et.al., 2021} \\
\hline & & & & Inhibitory activity. & \\
\hline 16 & 28.188 & 1.41 & Dotriacontane & Anti-fungal & Prakash et.al., 2021 \\
\hline 17 & 4.716 & 1.18 & Hexanal & Anti-fungal & $\begin{array}{l}\text { Shuaibing Zhang et.al., } \\
2021\end{array}$ \\
\hline 18 & 21.940 & 1.15 & Z,Z-6,27-Hexatriactontadien-2-one & - & - \\
\hline
\end{tabular}

Table-4: Metabolites from bioconverted DHA using Escherichia coli. 


\begin{tabular}{|c|c|c|c|c|c|}
\hline S.No & R.Time & $\%$ & Compound Name & Medicinal Properties & References \\
\hline 1 & 4.165 & 17.03 & Benzene, Methyl- & - & - \\
\hline \multirow[t]{2}{*}{2} & 15.766 & 10.84 & Phenol, 2,4-Bis(1,1-Dimethylethyl & Anti-fungal & Devi et.al., 2021 \\
\hline & & & & Anti-oxidant & Idih et.al., 2021 \\
\hline \multirow[t]{2}{*}{3} & 6.517 & 4.75 & Oxime-, methoxy-phenyl- & Pancreatic lipase & Anand et.al., 2021 \\
\hline & & & & Inhibitory activity. & \\
\hline 4 & 18.019 & 4.61 & Eicosane & Anti-microbial & Octarya et.al., 2021 \\
\hline \multirow[t]{2}{*}{5} & 9.401 & 4.43 & Cyclotrisiloxane, hexamethyl- & Anti-oxidant & Kanimozhi et.al., 2021 \\
\hline & & & & Anti-biofilm & \\
\hline 6 & 16.862 & 4.11 & Hexadecane & - & - \\
\hline \multirow[t]{2}{*}{7} & 12.619 & 3.28 & Dodecane, 2,6,11-trimethyl- & Anti-microbial & Octarya et.al., 2021 \\
\hline & & & & Anti-oxidant & Khalaf et.al., 2021 \\
\hline 8 & 14.351 & 3.12 & Tetradecane & - & - \\
\hline 9 & 19.115 & 3.03 & Heneicosane & Anti-inflammatory & Barda et.al., 2021 \\
\hline 10 & 4.460 & 2.65 & 3-Hexanone & - & - \\
\hline 11 & 22.308 & 2.62 & Octadecane & - & - \\
\hline 12 & 4.538 & 2.39 & 2-Hexanone & - & - \\
\hline 13 & 24.218 & 2.32 & 1H,5H-Cyclopropa[G][1, 2, 4]Triazolo[1,2-A] & - & - \\
\hline \multirow[t]{2}{*}{14} & 24.162 & 2.31 & Hexatriacontane & Anti-oxidant & Jamil et.al., 2021 \\
\hline & & & & Anti-microbial & \\
\hline 15 & 20.757 & 1.90 & 1-Butyl 2-(8-Methylnonyl) Phthalate \# & - & - \\
\hline 16 & 26.220 & 1.90 & Silikonfett Se30 (Grevels) & - & - \\
\hline 17 & 9.209 & 1.86 & Octane, 6-Ethyl-2-Methyl- & - & - \\
\hline 18 & 3.365 & 1.82 & Furan, tetrahydro-2,5-dimethyl- & - & - \\
\hline 19 & 24.112 & 1.50 & Cyclodecasiloxane, eicosamethyl- & Immunotherapeutic agent & Gullilat et.al., 2021 \\
\hline \multirow[t]{2}{*}{20} & 25.846 & 1.48 & Tetrapentacontane & Anti-microbial & Kerem et.al., 2019 \\
\hline & & & & Anti-Oxidant & Xuanji et.al., 2016 \\
\hline 21 & 12.282 & 1.41 & Benzene, 1,3-Bis(1,1-Dimethylethyl)- & Anti-cancer & Jenifer et.al., 2021 \\
\hline 22 & 11.521 & 1.38 & Dodecane & - & - \\
\hline 23 & 20.574 & 1.29 & 4-Pentyl-Cyclohexanecarboxylic Acid & - & - \\
\hline
\end{tabular}

Table-5: Metabolites from bioconverted DHA using Staphylococcus spp. 


\begin{tabular}{|c|c|c|c|c|c|}
\hline S.No & R.Time & Percentage \% & Compound Name & Medicinal Properties & References \\
\hline 1 & 28.162 & 7.18 & Decanedioic acid, bis(2-ethylhexyl) ester & Anti-fungal & Rafiq et.al., 2021 \\
\hline \multirow[t]{4}{*}{2} & 20.769 & 6.67 & n-Hexadecanoic acid & Anti-bacterial & Ansari et.al., 2021 \\
\hline & & & & Anti-fungal & \\
\hline & & & & Anti-biofilm & \\
\hline & & & & Anti-cancer & \\
\hline \multirow[t]{2}{*}{3} & 22.688 & 5.70 & Octadecanoic acid & Anti-bacterial & Daniels et.al., 2021 \\
\hline & & & & Anti-oxidant & \\
\hline 4 & 4.164 & 5.56 & Benzene, Methyl- & - & - \\
\hline 5 & 8.781 & 5.49 & 1-Hexanol, 2-Ethyl- & - & - \\
\hline \multirow[t]{2}{*}{6} & 15.772 & 5.11 & Phenol, 2,4-Bis(1,1-Dimethylethyl)- & Anti-fungal & Devi et.al., 2021 \\
\hline & & & & Anti-oxidant & Idih et.al., 2021 \\
\hline 7 & 23.653 & 4.90 & Ergosta-5,7,9(11),22-tetraen-3-ol, (3.beta.,22E)- & - & - \\
\hline \multirow[t]{2}{*}{8} & 24.174 & 3.72 & Tetrapentacontane & Anti-microbial & Kerem et.al., 2019 \\
\hline & & & & Anti-Oxidant & Xuanji et.al., 2016 \\
\hline 9 & 18.024 & 2.88 & Eicosane & Anti-Microbial & Octarya et.al., 2021 \\
\hline 10 & 16.866 & 2.68 & Hexadecane & - & - \\
\hline 11 & 28.368 & 2.56 & Squalene & - & - \\
\hline 12 & 15.499 & 2.17 & Hexadecane, 2,6,10,14-Tetramethyl- & - & - \\
\hline 13 & 19.120 & 2.06 & Heneicosane & Anti-inflammatory & Barda et.al., 2021 \\
\hline \multirow[t]{2}{*}{14} & 22.473 & 1.96 & Oleic Acid & Anti-tumor & Giulitt et.al, 2021 \\
\hline & & & & Anti-microbial & Ghavam et.al., 2021 \\
\hline \multirow[t]{2}{*}{15} & 12.623 & 1.90 & Dodecane, 2,6,11-trimethyl- & Anti-microbial & Octarya et.al., 2021 \\
\hline & & & & Anti-oxidant & Khalaf et.al., 2021 \\
\hline 16 & 14.356 & 1.88 & Tetradecane & - & - \\
\hline 17 & 24.115 & 1.67 & Cyclodecasiloxane, eicosamethyl- & Immunotherapeutic agent & Gullilat et.al., 2021 \\
\hline 18 & 6.999 & 1.48 & 1-Propene, 3,3-Dichloro- & - & - \\
\hline 19 & 25.824 & 1.48 & Bis(2-ethylhexyl) phthalate & - & - \\
\hline 20 & 22.314 & 1.44 & Hexatriacontane & Anti-microbial & Jamil et.al., 2021 \\
\hline \multirow[t]{2}{*}{21} & 22.421 & 1.44 & 10(E),12(Z)-Conjugated linoleic acid & Anti-carcinogen & Hornung et.al., 2005 \\
\hline & & & & Anti-Inflammatory & \\
\hline \multirow[t]{2}{*}{22} & 4.450 & 1.36 & 3-Hexanone & Anti-microbial & Khalaf et.al., 2021 \\
\hline & & & & Anti-oxidant & \\
\hline 23 & 4.525 & 1.20 & 2-Hexanone & - & - \\
\hline 24 & 20.411 & 1.15 & Hexadecanoic acid, methyl ester & Anti-oxidant & Ahamefula Sunday et.al., 2021 \\
\hline 25 & 24.814 & 1.13 & 1,16-Dibromohexadecane & - & - \\
\hline \multirow[t]{2}{*}{26} & 18.671 & 0.99 & Tetradecanoic acid & Anti-oxidant & Ji et.al., 2021 \\
\hline & & & & Anti-bacterial & \\
\hline 27 & 20.709 & 0.98 & $2,6,10,15,19,23-$ Hexamethyltetracosane & - & - \\
\hline 28 & 9.212 & 0.95 & Octane, 6-Ethyl-2-Methyl- & Anti-cancer & Jenifer et.al., 2021 \\
\hline 29 & 20.581 & 0.94 & 4-Pentyl-Cyclohexanecarboxylic Acid & - & - \\
\hline \multirow[t]{2}{*}{30} & 4.766 & 0.90 & 2-Hexanol & Antimicrobial & Javaid et.al., 2021 \\
\hline & & & & Anti-inflammatory & \\
\hline 31 & 16.065 & 0.88 & Hexadecane, 2,6,10,14-Tetramethyl & - & - \\
\hline
\end{tabular}




\begin{tabular}{|c|c|c|c|c|c|}
\hline S.No & R.Time & Percentage \% & Compound Name & Medicinal Properties & References \\
\hline 33 & 11.525 & 0.81 & Dodecane & - & - \\
\hline 34 & 16.778 & 0.81 & 1-Hexadecanol & - & - \\
\hline 35 & 4.691 & 0.76 & 3-Hexanol & Anti-bacterial & Rojas et.al., 2021 \\
\hline
\end{tabular}

Table-6: Metabolites from bioconverted DHA using Enterococcus spp

\begin{tabular}{|c|c|c|c|c|c|}
\hline S.No & R.Time & Percentage\% & Compound Name & Medicinal Properties & References \\
\hline \multirow[t]{4}{*}{1} & 20.803 & 15.04 & $\mathrm{n}$-Hexadecanoic acid & Anti-bacterial & Ansari et.al., 2021 \\
\hline & & & & Anti-fungal & \\
\hline & & & & Anti-biofilm & \\
\hline & & & & Anti-cancer & \\
\hline \multirow[t]{2}{*}{2} & 22.709 & 10.97 & Octadecanoic acid & Anti-bacterial & Daniels et.al., 2021 \\
\hline & & & & Anti-oxidant & \\
\hline 3 & 23.462 & 10.31 & Palmitoyl chloride & - & - \\
\hline \multirow[t]{2}{*}{4} & 24.962 & 8.77 & 9-Octadecenoic acid (Z)-, 2,3-dihydroxypropyl ester & Anti-microbial & Kumari et.al., 2021 \\
\hline & & & & Anti-fungal & Antonio et.al., 2021 \\
\hline 5 & 27.952 & 10.69 & Butyl 4,7,10,13,16,19-docosahexaenoate & - & - \\
\hline 6 & 23.874 & 7.70 & Glycidyl palmitate & - & - \\
\hline 7 & 25.364 & 5.43 & 9-Octadecenoic acid (Z)-, oxiranylmethyl ester & - & - \\
\hline 8 & 3.172 & 5.02 & Heptane & - & - \\
\hline \multirow[t]{2}{*}{9} & 22.488 & 8.71 & Oleic Acid & Anti-tumor & Giulitt et.al, 2021 \\
\hline & & & & Anti-microbial & Ghavam et.al., 2021 \\
\hline \multirow[t]{2}{*}{10} & 25.468 & 2.07 & Hexadecanoic acid, 2-hydroxy-1-(hydroxymethyl)ethy & Anti-fungal & Khan et.al., 2021 \\
\hline & & & & Anti-microbial & Kumari et.al., 2021 \\
\hline 11 & 27.861 & 2.73 & Methyl 4,7,10,13,16-docosapentaenoate & - & - \\
\hline 12 & 26.959 & 1.87 & cis-10-Pentadecenoic acid, isobutyl ester & - & - \\
\hline \multirow[t]{2}{*}{13} & 18.677 & 1.65 & Tetradecanoic acid & Anti-oxidant & Ji et.al., 2021 \\
\hline & & & & Anti-bacterial & \\
\hline \multirow[t]{2}{*}{14} & 25.163 & 1.59 & Octadecanoic acid, 2,3-dihydroxypropyl ester & Anti-microbial & Kumari et.al., 2021 \\
\hline & & & & Anti-oxidant & Vural et.al., 2021 \\
\hline \multirow[t]{2}{*}{15} & 15.771 & 1.50 & Phenol, 2,4-Bis(1,1-Dimethylethyl)- & Anti-fungal & Devi et.al., 2021 \\
\hline & & & & Anti-oxidant & Idih et.al., 2021 \\
\hline 16 & 22.429 & 1.46 & 9,12-Octadecadienoic Acid (Z,Z)- & Anti-bacterial & Jabbar et.al., 2021 \\
\hline 17 & 25.561 & 1.28 & Myristic acid glycidyl ester & - & - \\
\hline 19 & 18.023 & 0.80 & Eicosane & Anti-microbial & Octarya et.al., 2021 \\
\hline 20 & 16.866 & 0.75 & Hexadecane & - & - \\
\hline 21 & 24.915 & 0.61 & 3-(\{[2-(4-Fluorophenyl)Ethyl]Amino\}Methyl & - & - \\
\hline 22 & 19.118 & 0.59 & Heneicosane & Anti-inflammatory & Barda et.al., 2021 \\
\hline 23 & 12.623 & 0.48 & Dodecane, 4,6-dimethyl- & - & - \\
\hline
\end{tabular}

Table - 7: Antibacterial activity of Bioconverted DHA extract 


\begin{tabular}{|c|c|c|c|c|c|c|c|c|c|}
\hline \multirow[t]{2}{*}{ S.No. } & \multirow{2}{*}{$\begin{array}{l}\text { Clinical } \\
\text { Pathogens }\end{array}$} & \multicolumn{8}{|c|}{ Zone of Inhibition (mm) } \\
\hline & & $\begin{array}{l}\text { Bioconverted } \\
\text { DHA extract of } \\
\text { Bifidobacterium } \\
\text { spp. (90 }(9 \mathrm{~g} / \mathrm{mL})\end{array}$ & $\begin{array}{l}\text { Bioconverted } \\
\text { DHA extract of } \\
\text { Lactobacillus } \\
\text { spp. } \\
(90 \mu / \mathrm{mL})\end{array}$ & $\begin{array}{l}\text { Bioconverted } \\
\text { DHA extract } \\
\text { of Escherichia } \\
\text { coli } \\
(90 \mu \mathrm{g} / \mathrm{mL})\end{array}$ & $\begin{array}{l}\text { Bioconverted } \\
\text { DHA extract } \\
\text { of Citrobacter } \\
\text { spp. } \\
(90 \mu \mathrm{g} / \mathrm{mL})\end{array}$ & $\begin{array}{l}\text { Bioconverted } \\
\text { DHA extract } \\
\text { of } \\
\text { Enterobacter } \\
\text { spp. } \\
(90 \mu \mathrm{g} / \mathrm{mL})\end{array}$ & $\begin{array}{l}\text { Gentamycin } \\
(10 \mu \mathrm{g} / \mathrm{mL}) \\
\text { (Positive } \\
\text { Control) }\end{array}$ & DMSO & $\begin{array}{l}\text { Sterile } \\
\text { Distilled } \\
\text { water } \\
\text { (Negative } \\
\text { Control) }\end{array}$ \\
\hline
\end{tabular}

\begin{tabular}{|llllllll|}
\hline 1. & Bacillus cereus & 25 & 24 & 26 & 25 & 24 & 0 \\
\hline 2 & Escherichia coli & 15 & 16 & 14 & 15 & 17 & 0 \\
\hline 3. & $\begin{array}{l}\text { Pseudomonas } \\
\text { aeruginosa }\end{array}$ & 19 & 18 & 20 & 19 & 20 & 0 \\
\hline 4. & $\begin{array}{l}\text { Salmonella } \\
\text { enteritidis }\end{array}$ & 16 & 15 & 14 & 15 & 13 & 0 \\
\hline 5. & $\begin{array}{l}\text { Staphylococcus } \\
\text { aureus }\end{array}$ & 20 & 19 & 18 & 20 & 19 & 0 \\
\hline
\end{tabular}

Table - 8: Metabolites from Bioconverted DHA using Gut Bacteria 


\begin{tabular}{|c|c|c|c|c|c|}
\hline S.No & R.Time & $\begin{array}{l}\text { Percentage } \\
\%\end{array}$ & Compound Name & Medicinal Properties & References \\
\hline \multirow[t]{3}{*}{1} & 22.176 & 13.27 & 2-Nonadecanone & Anti-depression & Lee et.al., 2018 \\
\hline & & & & Anti-bacterial & Mihigo et.al., 2015 \\
\hline & & & & Anti-tumor & Figueiredo et.al., 2014 \\
\hline 2 & 21.941 & 12.91 & Z,Z-6,27-Hexatriactontadien-2-one & - & - \\
\hline \multirow[t]{3}{*}{3} & 43.881 & 11.48 & cis-9-Hexadecenal & Anti-biofilm & Hoda et.al., 2019 \\
\hline & & & & Anti-melanogenic & Hoda et.al., 2020 \\
\hline & & & & Anti-fungal & \\
\hline \multirow[t]{3}{*}{4} & 21.395 & 7.89 & Eicosanal- & Anti-depressant & Uddin et.al., 2021 \\
\hline & & & & Anxiolytic effects and & \\
\hline & & & & Anti-oxidant & \\
\hline 5 & 26.623 & 7.54 & Stigmast-5-En-3-OI, Oleat & Anti-obesity & Ghinmy et.al., 2019 \\
\hline \multirow[t]{2}{*}{6} & 26.922 & 10.27 & Tetrapentacontane & Anti-microbial & Kerem et.al., 2019 \\
\hline & & & & Anti-oxidant & Xuanji et.al., 2016 \\
\hline \multirow[t]{2}{*}{7} & 22.489 & 13.93 & Oleic Acid & Anti-tumor & Giulitt et.al, 2021 \\
\hline & & & & Anti-microbial & Ghavam et.al., 2021 \\
\hline \multirow[t]{2}{*}{8} & 22.698 & 32.53 & Octadecanoic acid & Anti-bacterial & Daniels et.al., 2021 \\
\hline & & & & Anti-oxidant & \\
\hline \multirow[t]{3}{*}{9} & 21.275 & 2.15 & (Z)-3-(Heptadec-10-en-1-yl)phenol & Anti-bacterial & Van et.al., 2020 \\
\hline & & & & Anti-diarrheal & Nemkul et.al., 2021 \\
\hline & & & & Anti-oxidant & \\
\hline 10 & 20.193 & 2.69 & 2-Heptadecanone & & \\
\hline \multirow[t]{4}{*}{11} & 20.774 & 34.68 & n-Hexadecanoic acid & Anti-bacterial & Ansari et.al., 2021 \\
\hline & & & & Anti-fungal & \\
\hline & & & & Anti-biofilm & \\
\hline & & & & Anti-cancer & \\
\hline 12 & 24.730 & 7.63 & Heneicosane & Anti-inflammatory & Barda et.al., 2021 \\
\hline \multirow[t]{2}{*}{13} & 15.782 & 31.59 & Phenol, 2,4-Bis(1,1-Dimethylethyl)- & Anti-fungal & Devi et.al., 2021 \\
\hline & & & & Anti-oxidant & Idih et.al., 2021 \\
\hline 14 & 19.326 & 1.64 & Hexadecanal & - & - \\
\hline 15 & 21.880 & 1.34 & 8,11-Heptadecadienal, (8Z,11Z)- & - & - \\
\hline 16 & 21.102 & 1.28 & 9,17-Octadecadienal, (Z)- & Antimicrobial agent & Insanu et.al., 2021 \\
\hline 17 & 23.985 & 0.99 & 2-Pentacosanone & - & - \\
\hline 18 & 16.871 & 8.53 & Hexadecane & - & - \\
\hline 19 & 27.063 & 0.97 & 8-Octadecanone & Antimicrobial metabolite & Tayung et.al., 2011 \\
\hline 20 & 25.397 & 0.89 & Stigmasta-5,22-Dien-3-OI, Acetat, (3-Beta,2 & Antimicrobial & Richa Bhardwaj 2018 \\
\hline 21 & 28.040 & 0.72 & 9-Heptadecanone & - & - \\
\hline 22 & 27.786 & 0.70 & Z,Z-6,28-Heptatriactontadien-2-one & $\begin{array}{l}\text { Alpha-amylase Inhibition And Antioxidant } \\
\text { Activity }\end{array}$ & Unnikrishnan et.al., 2015 \\
\hline \multirow[t]{2}{*}{23} & 27.000 & 17.11 & 9-Octadecenoic acid (Z)-, 2,3-dihydroxypropyl & Anti-microbial & Kumari et.al., 2021 \\
\hline & & & & Anti-fungal & Antonio et.al., 2021 \\
\hline \multirow[t]{2}{*}{24} & 27.214 & 8.7 & Octadecanoic acid, 2,3-dihydroxypropyl ester & Anti-microbial & Kumari et.al., 2021 \\
\hline & & & & Anti-oxidant & Vural et.al., 2021 \\
\hline 25 & 25.389 & 9.83 & $\begin{array}{l}\text { 9-Octadecenoic acid (Z)-, oxiranylmethyl } \\
\text { ester }\end{array}$ & - & - \\
\hline
\end{tabular}




\begin{tabular}{|c|c|c|c|c|c|}
\hline S.No & R.Time & $\begin{array}{l}\text { Percentage } \\
\%\end{array}$ & Compound Name & Medicinal Properties & References \\
\hline 26 & 26.680 & 4.30 & n-Propyl 9-octadecenoate & - & - \\
\hline 27 & 25.582 & 3.50 & Glycidyl palmitate & Anti-staphylococcal activity & Houdkova et.al., 2021 \\
\hline \multirow[t]{2}{*}{28} & \multirow[t]{2}{*}{25.490} & \multirow[t]{2}{*}{4.9} & \multirow{2}{*}{$\begin{array}{l}\text { Hexadecanoic acid, 2-hydroxy-1- } \\
\text { (hydroxymethyl)ethy }\end{array}$} & Anti-fungal & Khan et.al., 2021 \\
\hline & & & & Anti-microbial & Kumari et.al., 2021 \\
\hline 29 & 26.786 & 2.31 & 1-Cyclohexyldimethylsilyloxybutane & - & - \\
\hline 30 & 26.880 & 2.26 & Octadecanoic acid, propyl ester & - & - \\
\hline 31 & 24.491 & 1.75 & Eicosanoic acid & Anti-bacterial & Pavani et.al., 2021 \\
\hline \multirow[t]{2}{*}{32} & \multirow[t]{2}{*}{6.560} & \multirow[t]{2}{*}{6.18} & \multirow[t]{2}{*}{ Oxime-, methoxy-phenyl- } & Pancreatic lipase & \multirow[t]{2}{*}{ Anand et.al., 2021} \\
\hline & & & & Inhibitory activity. & \\
\hline 33 & 28.188 & 1.41 & Dotriacontane & Anti-fungal & Prakash et.al., 2021 \\
\hline 34 & 4.716 & 1.18 & Hexanal & Anti-fungal & $\begin{array}{l}\text { Shuaibing Zhang et.al., } \\
2021\end{array}$ \\
\hline 35 & 4.165 & 22.59 & Benzene, Methyl- & - & - \\
\hline 36 & 18.019 & 8.29 & Eicosane & Anti-microbial & Octarya et.al., 2021 \\
\hline \multirow[t]{2}{*}{37} & \multirow[t]{2}{*}{9.401} & \multirow[t]{2}{*}{4.43} & \multirow[t]{2}{*}{ Cyclotrisiloxane, hexamethyl- } & Anti-oxidant & \multirow[t]{2}{*}{ Kanimozhi et.al., 2021} \\
\hline & & & & Anti-biofilm & \\
\hline \multirow[t]{2}{*}{38} & \multirow[t]{2}{*}{12.619} & \multirow[t]{2}{*}{5.18} & \multirow[t]{2}{*}{ Dodecane, 2,6,11-trimethyl- } & Anti-microbial & Octarya et.al., 2021 \\
\hline & & & & Anti-oxidant & Khalaf et.al., 2021 \\
\hline 39 & 14.351 & 5.0 & Tetradecane & - & - \\
\hline \multirow[t]{2}{*}{40} & \multirow[t]{2}{*}{4.460} & \multirow[t]{2}{*}{4.01} & \multirow[t]{2}{*}{ 3-Hexanone } & Anti-microbial & \multirow[t]{2}{*}{ Khalaf et.al., 2021} \\
\hline & & & & Anti-oxidant & \\
\hline 41 & 22.308 & 2.62 & Octadecane & - & - \\
\hline 42 & 4.538 & 3.59 & 2-Hexanone & - & - \\
\hline 43 & 24.218 & 2.32 & 1H,5H-Cyclopropa[G][1, 2, 4] Triazolo[1,2-A] & - & - \\
\hline \multirow[t]{2}{*}{44} & \multirow[t]{2}{*}{24.162} & 3.75 & Hexatriacontane & Anti-oxidant & Jamil et.al., 2021 \\
\hline & & & & Anti-microbial & \\
\hline 45 & 20.757 & 1.90 & 1-Butyl 2-(8-Methylnonyl) Phthalate \# & - & - \\
\hline 46 & 26.220 & 1.90 & Silikonfett Se30 (Grevels) & - & - \\
\hline 47 & 9.209 & 1.86 & Octane, 6-Ethyl-2-Methyl- & - & - \\
\hline 48 & 3.365 & 1.82 & Furan, tetrahydro-2,5-dimethyl- & - & - \\
\hline 49 & 24.112 & 1.50 & Cyclodecasiloxane, eicosamethyl- & Immunotherapeutic agent & Gullilat et.al., 2021 \\
\hline 50 & 12.282 & 1.41 & Benzene, 1,3-Bis(1,1-Dimethylethyl)- & Anti-cancer & Jenifer et.al., 2021 \\
\hline 51 & 11.521 & 2.19 & Dodecane & - & - \\
\hline 52 & 20.574 & 1.29 & 4-Pentyl-Cyclohexanecarboxylic Acid & - & - \\
\hline 53 & 28.162 & 7.18 & Decanedioic acid, bis(2-ethylhexyl) ester & Anti-fungal & Rafiq et.al., 2021 \\
\hline 54 & 8.781 & 5.49 & 1-Hexanol, 2-Ethyl- & - & - \\
\hline 55 & 23.653 & 4.90 & $\begin{array}{l}\text { Ergosta-5,7,9(11),22-tetraen-3-ol, } \\
\text { (3.beta.,22E)- }\end{array}$ & - & - \\
\hline 56 & 28.368 & 2.56 & Squalene & - & - \\
\hline 57 & 15.499 & 3.05 & Hexadecane, 2,6,10,14-Tetramethyl- & - & - \\
\hline 58 & 24.115 & 1.67 & Cyclodecasiloxane, eicosamethyl- & Immunotherapeutic agent & Gullilat et.al., 2021 \\
\hline 59 & 6.999 & 1.48 & 1-Propene, 3,3-Dichloro- & - & - \\
\hline 60 & 25.824 & 1.48 & Bis(2-ethylhexyl) phthalate & - & - \\
\hline
\end{tabular}




\begin{tabular}{|c|c|c|c|c|c|}
\hline S.No & R.Time & $\begin{array}{l}\text { Percentage } \\
\%\end{array}$ & Compound Name & Medicinal Properties & References \\
\hline \multirow[t]{2}{*}{61} & 22.421 & 1.44 & 10(E),12(Z)-Conjugated linoleic acid & Anti-carcinogen & Hornung et.al., 2005 \\
\hline & & & & Anti-Inflammatory & \\
\hline 62 & 20.411 & 1.15 & Hexadecanoic acid, methyl ester & Anti-oxidant & $\begin{array}{l}\text { Ahamefula Sunday et.al., } \\
2021\end{array}$ \\
\hline 63 & 24.814 & 1.13 & 1,16-Dibromohexadecane & - & - \\
\hline \multirow[t]{2}{*}{64} & 18.671 & 5.89 & Tetradecanoic acid & Anti-oxidant & Ji et.al., 2021 \\
\hline & & & & Anti-bacterial & \\
\hline 65 & 20.709 & 0.98 & $2,6,10,15,19,23$-Hexamethyltetracosane & - & - \\
\hline 66 & 9.212 & 0.95 & Octane, 6-Ethyl-2-Methyl- & Anti-cancer & Jenifer et.al., 2021 \\
\hline 67 & 20.581 & 0.94 & 4-Pentyl-Cyclohexanecarboxylic Acid & - & - \\
\hline 68 & 4.766 & 0.90 & 2-Hexanol & Antimicrobial Antiinflammatory & Javaid et.al., 2021 \\
\hline 69 & 12.730 & 0.85 & 4-Propylbenzaldehyde & - & - \\
\hline 70 & 16.778 & 0.81 & 1-Hexadecanol & - & - \\
\hline 71 & 4.691 & 0.76 & 3-Hexanol & Anti-bacterial & Rojas et.al., 2021 \\
\hline 72 & 13.282 & 0.75 & Dodecane, 4,6-dimethyl- & - & - \\
\hline 73 & 23.462 & 10.31 & Palmitoyl chloride & - & - \\
\hline 74 & 27.952 & 10.69 & Butyl 4,7,10,13,16,19-docosahexaenoate & - & - \\
\hline 75 & 23.874 & 7.70 & Glycidyl palmitate & - & - \\
\hline 76 & 3.172 & 5.02 & Heptane & - & - \\
\hline 77 & 27.861 & 2.73 & Methyl 4,7,10,13,16-docosapentaenoate & - & - \\
\hline 78 & 26.959 & 1.87 & cis-10-Pentadecenoic acid, isobutyl ester & - & - \\
\hline 79 & 22.429 & 1.46 & 9,12-Octadecadienoic Acid (Z,Z)- & Anti-bacterial & Jabbar et.al., 2021 \\
\hline 80 & 25.561 & 1.28 & Myristic acid glycidyl ester & - & - \\
\hline 81 & 24.915 & 0.61 & 3-(\{[2-(4-Fluorophenyl)Ethyl]Amino\}Methyl & - & - \\
\hline 82 & 12.623 & 0.48 & Dodecane, 4,6-dimethyl- & - & - \\
\hline
\end{tabular}

\section{Figures}




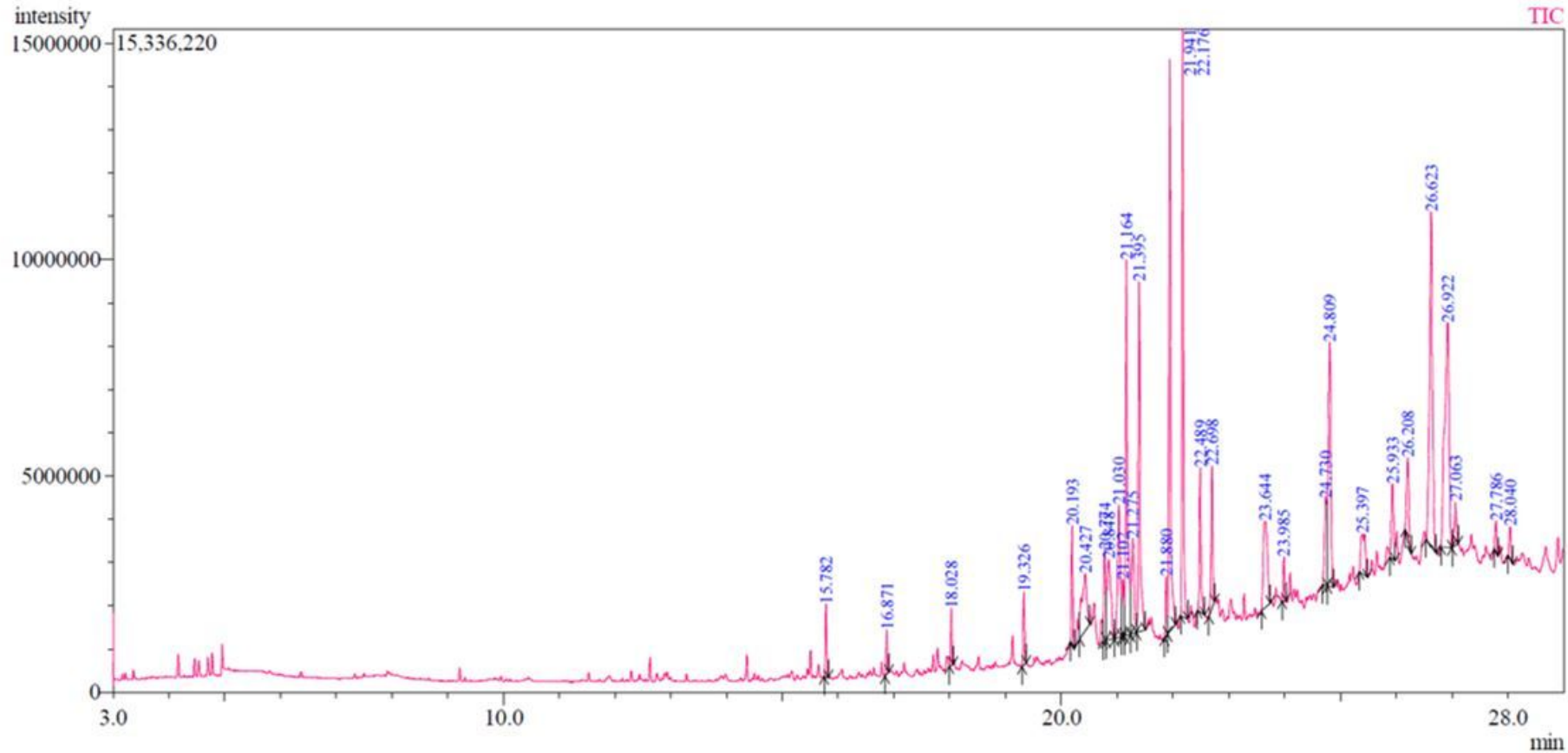

Figure 1

GC-MS analysis of bioconverted DHA metabolites 